\title{
Characterization of bariatric surgery and outcomes using administrative claims data in the research network of a nationwide commercial health plan
}

\author{
Qinli Ma ${ }^{1 *}$ (D) Michael Mack', Sonali Shambhu' ${ }^{1}$ Kathleen McTigue ${ }^{2}$ and Kevin Haynes ${ }^{1}$
}

\begin{abstract}
Background: The supplementation of electronic health records data with administrative claims data may be used to capture outcome events more comprehensively in longitudinal observational studies. This study investigated the utility of administrative claims data to identify outcomes across health systems using a comparative effectiveness study of different types of bariatric surgery as a model.

Methods: This observational cohort study identified patients who had bariatric surgery between 2007 and 2015 within the HealthCore Anthem Research Network (HCARN) database in the National Patient-Centered Clinical Research Network (PCORnet) common data model. Patients whose procedures were performed in a member facility affiliated with PCORnet Clinical Research Networks (CRNs) were selected. The outcomes included a 30-day composite adverse event (including venous thromboembolism, percutaneous/operative intervention, failure to discharge and death), and all-cause hospitalization, abdominal operation or intervention, and in-hospital death up to 5 years after the procedure. Outcomes were classified as occurring within or outside PCORnet CRN health systems using facility identifiers.

Results: We identified 4899 patients who had bariatric surgery in one of the PCORnet CRN health systems. For 30-day composite adverse event, the inclusion of HCARN multi-site claims data marginally increased the incidence rate based only on HCARN single-site claims data for PCORnet CRNs from 3.9 to $4.2 \%$. During the 5 -year follow-up period, $56.8 \%$ of all-cause hospitalizations, 31.2\% abdominal operations or interventions, and 32.3\% of in-hospital deaths occurred outside PCORnet CRNs. Incidence rates (events per 100 patient-years) were significantly lower when based on claims from a single PCORnet CRN only compared to using claims from all health systems in the HCARN: all-cause hospitalization, 11.0 (95\% Confidence Internal [Cl]: 10.4, 11.6) to 25.3 (95\% Cl: 24.4, 26.3); abdominal operations or interventions, 4.2 (95\% Cl: 3.9, 4.6) to 6.1 (95\% Cl: 5.7, 6.6); in-hospital death, 0.2 (95\% Cl: $0.11,0.27)$ to 0.3 (95\% Cl: $0.19,0.38)$.

(Continued on next page)
\end{abstract}

\footnotetext{
*Correspondence: ama@healthcore.com

${ }^{1}$ Translational Research for Affordability and Quality, HealthCore, Inc, Wilmington, DE, USA

Full list of author information is available at the end of the article
}

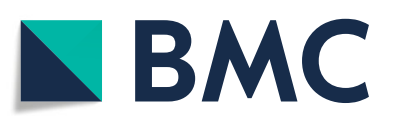

(- The Author(s). 2021 Open Access This article is licensed under a Creative Commons Attribution 4.0 International License, which permits use, sharing, adaptation, distribution and reproduction in any medium or format, as long as you give appropriate credit to the original author(s) and the source, provide a link to the Creative Commons licence, and indicate if changes were made. The images or other third party material in this article are included in the article's Creative Commons licence, unless indicated otherwise in a credit line to the material. If material is not included in the article's Creative Commons licence and your intended use is not permitted by statutory regulation or exceeds the permitted use, you will need to obtain permission directly from the copyright holder. To view a copy of this licence, visit http://creativecommons.org/licenses/by/4.0/. The Creative Commons Public Domain Dedication waiver (http://creativecommons.org/publicdomain/zero/1.0/) applies to the data made available in this article, unless otherwise stated in a credit line to the data. 
(Continued from previous page)

Conclusions: Short-term inclusion of multi-site claims data only marginally increased the incidence rate computed from single-site claims data alone. Longer-term follow up captured a notable number of events outside of PCORnet CRNs. The findings suggest that supplementing claims data improves the outcome ascertainment in longitudinal observational comparative effectiveness studies.

Keywords: Administrative claims data, Electronic health records data, Data integration, Bariatric surgery, PCORnet Clinical Research Networks

\section{Background}

Electronic health records (EHR) data, with rich medical history and clinical information, is a real-world resource for conducting comparative effectiveness research [1]. However, most EHR systems keep medial encounters of a patient under a particular health care delivery system and are often challenged by incomplete data [2]. Reliance on EHR data from a single health system, however, could result in only partial capture of data across patients' healthcare journeys, particularly when patients receive care from multiple health systems [3-7]. This presents challenges when studying long-term outcomes presenting greater concern for missing outcome events and biasing results.

For a more complete picture, a potential cost-efficient approach is to supplement EHR data with health insurance administrative claims data. Administrative claims data contains members' health services information across health systems over defined enrollment periods. In fact, the use of claims data in comparative effectiveness studies is a reliable source of real-world data [8-11]. However, the value of supplementing claims data in ascertaining outcome events in longitudinal comparative effectiveness studies is not well quantified. One barrier is that integration of data from different sources with inconsistent quality exacerbates the difficulties of building complete longitudinal observational data sets [12-16].

Representing a new research paradigm, the PatientCentered Outcomes Research Institute (PCORI) launched the National Patient-Centered Clinical Research Network (PCORnet) in 2014 in an effort to help address these challenges. The network leverages the power of health data and reusable research infrastructures from 13 Clinical Research Networks (CRNs) and two Health Plan Research Networks (HPRNs) to support multi-institutional research. Each CRN within PCORnet comprises health systems including hospitals, integrated delivery systems, and federally qualified health centers. Each HPRN is affiliated with a national health insurance provider. All CRNs and HPRNs store their EHR data and/or claims data in a common data format (known as PCORnet Common Data Model) that can be queried by researchers across institutions [17, 18]. Collectively, PCORnet includes more than 60 million geographically diverse patients, and is one of the largest and most representative healthcare research consortia in the United States.

One of the three PCORnet demonstration projects was the retrospective comparative effectiveness study on the three commonly performed types of weight loss surgery, adjustable gastric banding (AGB), Roux-en-Y gastric bypass (RYGB), and sleeve gastrectomy (SG) [19]. The study assessed the 5-year risks and benefits of bariatric surgical procedures in various populations [20-24]. This topic was selected as exemplary by PCORI firstly due to the increasing prevalence of obesity in the United States (with ageadjusted prevalence of $42.4 \%$ in adults in 2017-2018 [25]), and secondly due to the lack of evidence for SG. This procedure has less than a decade history in the country while it has quickly become the most commonly performed. The findings, particularly comparison of SG and bypass will enable patients and healthcare providers in making better-informed decision. The wide scope of data in PCORnet had the potential for large sample sizes that are essential to evaluate weight loss and rare adverse outcomes overtime [24].

Taking the PCORnet bariatric study as a model [22, 24], the objective of this study was to evaluate the utility of claims data in supplementing data available only to specific health systems (contained in EHR systems) in capturing outcomes longitudinally across health systems. We quantied the value of supplementing EHR data with claims data in outcome ascertainment in a large comparative effectiveness observational study with up-to 5 years follow-up. The claims data is from the HealthCore Anthem Research Network (HCARN), a participating PCORnet health plan that extracts administrative claims data from a large United States-based insurance company for inclusion in the PCORnet common data model. This study utilized claims data from HCARN to replicate the data a single health system (EHR data) would have available and compared outcome ascertainment limiting to data from a single health system to ascertainment utilizing complete claims profile across health systems.

\section{Methods}

Study design and data source

Eligible patients were identified via a computerized query for PCORnet bariatric computable phenotypes 
within the HCARN data stored in the PCORnet common data model (CDM). The HealthCore Intergraded Research Database (HIRD) is a repository of longitudinal claims data extracted, transformed, and loaded into the PCORnet CDM for HCARN enrollees, and is representative of the commercially insured population in the United States. Patient-level information for provider and facility visits in the HIRD were used to characterize care across healthcare delivery systems. HCARN members who had overlapping membership in a PCORnet CRN health system based on institutional claims for bariatric surgery were identified. Adverse events of interest for the overlapping patients in the claims data (based on outcome events in the PCORnet Bariatric Study [24]) were categorized as occurring either inside or outside PCORnet index CRN health systems. This study, which used a limited deidentified dataset, received an exemption from informed consent requirements by the New England Independent Review Board.

\section{Study population}

The study population consisted of HCARN members who satisfied the criteria established for the PCORnet Bariatric Study using the 2016 version of the computable phenotype [12]. That is, patients who had one of the three common bariatric surgery procedures during inpatient or ambulatory care - AGB, RYGB or SG from 01/01/2007 to 09/30/2015 (see Additional file 1: Appendix Table 1 for International Classification of Diseases-9 (ICD-9) procedure codes and Current Procedural Terminology (CPT) codes used to identify bariatric procedures). The first procedure date was defined as the index date for each patient.

To be included, patients were required to be $\leq 79$ years old at the index date. In addition, one year of continuous medical eligibility in a health plan prior to the index date was required for baseline evaluations. Patients with multiple conflicting bariatric procedure codes such as any revisional bariatric procedure code, gastrointestinal cancer diagnosis code, or fundoplasty in the year before the index procedure were excluded. Also excluded were members who had emergency department encounters on the day of the index bariatric procedure.

Post-exclusion, patients who had a bariatric surgery of interest at any PCORnet CRN health systems (based on institutional identifiers) were identified and included in the analysis. The health system where a patient had bariatric surgery was defined as the index CRN health system for that patient.

\section{Outcome events of interest}

The main outcome measures, derived from the PCORnet Bariatric study, [12, 19, 22, 24] were safety outcomes requiring medical attention. The short-term outcome measure was a 30-day composite adverse event, including venous thromboembolism, percutaneous, operative, or endoscopic intervention, failure to discharge and death. The long-term outcome measures included major abdominal operation or intervention, all-cause hospitalization, and in-hospital death up to 5 years after bariatric surgery. For exploratory purposes, outcome measures that occurred within 1 year and 3 years post bariatric surgery were also examined to evaluate different follow-up times and the impact on outcome identification. In addition, death information was retrieved from the Social Security Administration, hospital discharge status in medical claims, and health plan enrollment file to evaluate overall mortality. Venous thromboembolism were identified by ICD-9 diagnosis codes, and abdominal operations/interventions were defined by ICD-9 procedure codes and CPT codes during a hospital stay. Patient follow-up was from the index date to the first of the following: end date of study, 09/ $30 / 2015$; date of death, or to the cessation of health plan enrollment, and up to 1 year, 3 years and 5 years respectively.

Outcome events that occurred during the follow-up period were attributed to two exclusive categories in accordance with institutional identifiers linked to the hospital stay: within and outside the index CRN health system.

\section{Statistical analysis}

Patients' demographic characteristics, including age, gender, and region, were examined during the baseline period. Descriptive statistics were used to report outcome events that occurred within the index CRN health system to those that occurred outside CRN health systems. Incidence rates (per 100 patient-years) were reported respectively for 1) a single index health system, for which only the claims from a PCORnet health system were examined; 2) all health systems in the HCAR $\mathrm{N}$, for which full administrative claims data in HCARN were examined.

\section{Results \\ Patient disposition}

A total of 112,089 bariatric surgery patients were identified in the overall HCARN cohort during the study period, as shown in Fig. 1. Upon applying age, enrollment, and clinical requirements, a total of 67,915 patients were selected. Among them, 4899 had an index bariatric surgery performed at a PCORnet CRN health system. Surgeries occurred in 66 health systems across 11 CRNs ranging from 27 to 1613 procedures (Additional file 1: Appendix Table 2).

The 4899 patients had a mean age of 44.6 years, was $70.5 \%$ female, and had average follow-up time of 28 


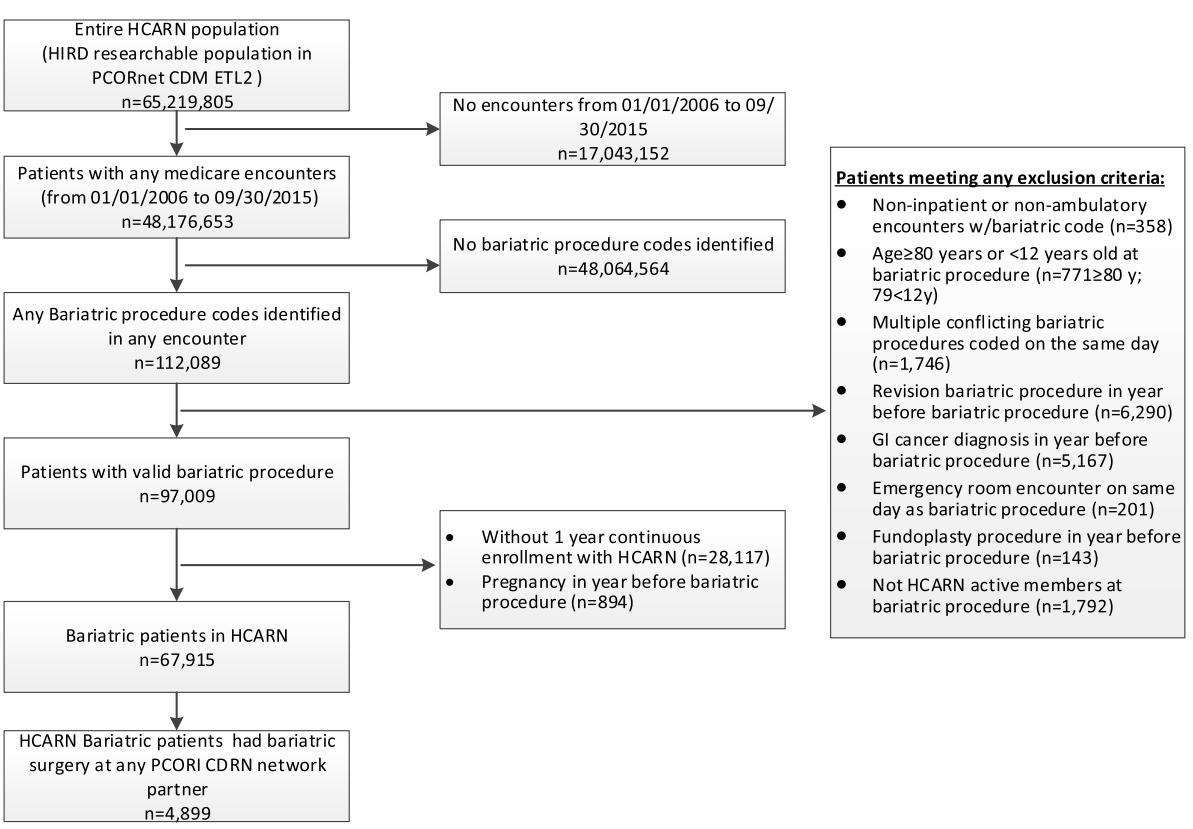

Fig. 1 Patient attrition

months (median $=23$ months). The Northeast and South regions each had slightly more than one third of the patients. The most commonly occurring comorbid conditions overall were hypertension (55.9\%), diabetes mellitus (48.5\%), dyslipidemia (43.1\%), gastroesophageal reflux (31.1\%), sleep apnea (26.4\%), and depression (15.8\%); mean (SD) Mixed Comorbidity Index score was 0.2 (1.13). About 34.3 and $39.4 \%$ patients received RYGB and AGB respectively, while $26.3 \%$ had SG procedures. From 2007 to 2015, a peak in the proportions of patients with AGB was observed in 2009 followed by a substantial decline afterwards. The proportion undergoing SG increased substantially and the trend for RYGB was relatively stable. The RYGB patients were slightly older, likelier to be female, and had more comorbid conditions, as shown in Table 1.

\section{0-day composite adverse events}

Among 4899 patients, 4752 continuously enrolled with their health plan for the 30 days after the index date, and therefore were included in the analysis of 30-day adverse events. Using HCARN multi-site claims data marginally impacted the incidence rate of using HCARN single-site claims data for index CRN health system, an increase from 3.9 to $4.2 \%$, as shown in Table 2. A large majority of patients, 162 of 174 (93.1\%), had their percutaneous or endoscopic intervention performed at the hospital where they received their index bariatric procedure, however, only 15 out of 19 (78.9\%) had their venous thromboembolism treated at the index health system.
In an exploratory analysis, we examined the $15 \mathrm{pa}$ tients who were admitted to non-CRN hospitals for adverse events within 30 days. These 15 patients on average lived further away from the index CRN health system (average 55 miles, range from 6 miles to 142 miles at the zip code level) than from the hospital which treated the adverse outcome (average of 16 miles, range from 4 to 36 miles at zip code level).

\section{Long-term outcome measures}

Across health systems using all available HCAR N claims, slightly more than a quarter of the patients, 1361 (27.8\%), were admitted to hospitals, 540 (11.0\%) had abdominal operations/interventions, and $31(0.6 \%)$ died while in hospital up to 5 years after the bariatric surgery, as shown in Table 3. Upon the inclusion of all adverse events during the follow-up period, allowing for multiple admissions per patient, for the 4899 patients, there were 2893 hospital admissions, among which 701 were for abdominal operations or interventions.

When examining individuals, 3538 (72.2\%) patients had no hospitalization over the 5-year follow-up period. For those with hospital admission, 810 (16.5\%) had 1 hospitalization in which 415 (51.2\%) were admitted to the index CRN health system, that is, where the initial bariatric surgery was performed; 277 (5.6\%) patients had two hospitalizations, in which 111 (40.2\%) had both admissions in index health system and 60 (21.7\%) had one admission in the index health system. Among 275 patients who had three or more hospital admissions, 55 (20.0\%) had all admissions in index health system and 
Table 1 Demographic and clinical characteristics at baseline

\begin{tabular}{|c|c|c|c|c|c|c|c|c|}
\hline & \multicolumn{2}{|l|}{ All } & \multirow{2}{*}{$\begin{array}{l}\text { AGB } \\
\text { n/mean }\end{array}$} & \multirow[b]{2}{*}{$\% / S D$} & \multirow{2}{*}{$\begin{array}{l}\text { RYGB } \\
\text { n/mean }\end{array}$} & \multirow[b]{2}{*}{$\% / S D$} & \multirow{2}{*}{$\begin{array}{l}\text { SG } \\
\text { n/mean }\end{array}$} & \multirow[b]{2}{*}{$\% / S D$} \\
\hline & $\mathrm{n} / \mathrm{mean}$ & $\% / S D$ & & & & & & \\
\hline Number of patients, $\mathrm{n}(N=4899)$ & 4899 & $100.0 \%$ & 1681 & $34.3 \%$ & 1932 & $39.4 \%$ & 1286 & $26.3 \%$ \\
\hline Age, mean (SD) & 44.61 & 12.53 & 44.42 & 12.72 & 45.35 & 12.38 & 43.75 & 12.47 \\
\hline \multicolumn{9}{|l|}{ Age category, n (\%) } \\
\hline$<20$ & 114 & $2.3 \%$ & 33 & $2.0 \%$ & 39 & $2.0 \%$ & 42 & $3.3 \%$ \\
\hline $20-64$ & 4561 & $93.1 \%$ & 1567 & $93.2 \%$ & 1792 & $92.8 \%$ & 1202 & $93.5 \%$ \\
\hline $65-79$ & 224 & $4.6 \%$ & 81 & $4.8 \%$ & 101 & $5.2 \%$ & 42 & $3.3 \%$ \\
\hline Female, n (\%) & 3456 & $70.5 \%$ & 1152 & $68.5 \%$ & 1394 & $72.2 \%$ & 910 & $70.8 \%$ \\
\hline \multicolumn{9}{|l|}{ Geographic region (Census Bureau), n (\%) } \\
\hline Northeast & 1667 & $34.0 \%$ & 684 & $40.7 \%$ & 465 & $24.1 \%$ & 518 & $40.3 \%$ \\
\hline Midwest & 929 & $19.0 \%$ & 188 & $11.2 \%$ & 551 & $28.5 \%$ & 190 & $14.8 \%$ \\
\hline West & 677 & $13.8 \%$ & 89 & $5.3 \%$ & 306 & $15.8 \%$ & 282 & $21.9 \%$ \\
\hline South & 1624 & $33.1 \%$ & 719 & $42.8 \%$ & 609 & $31.5 \%$ & 296 & $23.0 \%$ \\
\hline Medicare coverage, n (\%) & 175 & $3.6 \%$ & 61 & $3.6 \%$ & 88 & $4.6 \%$ & 26 & $2.0 \%$ \\
\hline \multicolumn{9}{|l|}{ Year of index surgery, $\mathrm{n}(\%)$} \\
\hline 2007 & 388 & $7.9 \%$ & 230 & $13.7 \%$ & 148 & $7.7 \%$ & 10 & $0.8 \%$ \\
\hline 2008 & 442 & $9.0 \%$ & 280 & $16.7 \%$ & 151 & $7.8 \%$ & 11 & $0.9 \%$ \\
\hline 2009 & 528 & $10.8 \%$ & 331 & $19.7 \%$ & 192 & $9.9 \%$ & $<10$ & \\
\hline 2010 & 638 & $13.0 \%$ & 326 & $19.4 \%$ & 278 & $14.4 \%$ & 34 & $2.6 \%$ \\
\hline 2011 & 718 & $14.7 \%$ & 258 & $15.3 \%$ & 323 & $16.7 \%$ & 137 & $10.7 \%$ \\
\hline 2012 & 599 & $12.2 \%$ & 104 & $6.2 \%$ & 282 & $14.6 \%$ & 213 & $16.6 \%$ \\
\hline 2013 & 575 & $11.7 \%$ & 62 & $3.7 \%$ & 232 & $12.0 \%$ & 281 & $21.9 \%$ \\
\hline 2014 & 619 & $12.6 \%$ & 57 & $3.4 \%$ & 208 & $10.8 \%$ & 354 & $27.5 \%$ \\
\hline 2015 & 392 & $8.0 \%$ & 33 & $2.0 \%$ & 118 & $6.1 \%$ & 241 & $18.7 \%$ \\
\hline \multicolumn{9}{|l|}{ Comorbid conditions, ${ }^{*}$ (\%) } \\
\hline Diabetes mellitus & 2376 & $48.5 \%$ & 814 & $48.4 \%$ & 946 & $49.0 \%$ & 616 & $47.9 \%$ \\
\hline Gastroesophageal reflux & 1525 & $31.1 \%$ & 444 & $26.4 \%$ & 699 & $36.2 \%$ & 382 & $29.7 \%$ \\
\hline Hypertension & 2737 & $55.9 \%$ & 898 & $53.4 \%$ & 1147 & $59.4 \%$ & 692 & $53.8 \%$ \\
\hline Sleep apnea & 1291 & $26.4 \%$ & 275 & $16.4 \%$ & 593 & $30.7 \%$ & 423 & $32.9 \%$ \\
\hline Deep vein thromboembolism & 56 & $1.1 \%$ & 19 & $1.1 \%$ & 18 & $0.9 \%$ & 19 & $1.5 \%$ \\
\hline Pulmonary embolism & 55 & $1.1 \%$ & 21 & $1.2 \%$ & 17 & $0.9 \%$ & 17 & $1.3 \%$ \\
\hline Dyslipidemia & 2111 & $43.1 \%$ & 742 & $44.1 \%$ & 862 & $44.6 \%$ & 507 & $39.4 \%$ \\
\hline Anxiety & 456 & $9.3 \%$ & 106 & $6.3 \%$ & 204 & $10.6 \%$ & 146 & $11.4 \%$ \\
\hline Depression & 776 & $15.8 \%$ & 189 & $11.2 \%$ & 367 & $19.0 \%$ & 220 & $17.1 \%$ \\
\hline Eating disorder & 137 & $2.8 \%$ & 27 & $1.6 \%$ & 55 & $2.8 \%$ & 55 & $4.3 \%$ \\
\hline Kidney disease & 122 & $2.5 \%$ & 30 & $1.8 \%$ & 50 & $2.6 \%$ & 42 & $3.3 \%$ \\
\hline Non-alcoholic fatty liver disease. & 193 & $3.9 \%$ & 35 & $2.1 \%$ & 89 & $4.6 \%$ & 69 & $5.4 \%$ \\
\hline Osteoarthritis & 578 & $11.8 \%$ & 198 & $11.8 \%$ & 236 & $12.2 \%$ & 144 & $11.2 \%$ \\
\hline Polycystic ovarian syndrome & 149 & $3.0 \%$ & 43 & $2.6 \%$ & 60 & $3.1 \%$ & 46 & $3.6 \%$ \\
\hline Psychotic disorder & 433 & $8.8 \%$ & 108 & $6.4 \%$ & 196 & $10.1 \%$ & 129 & $10.0 \%$ \\
\hline Substance use disorder & 31 & $0.6 \%$ & $<10$ & & 17 & $0.9 \%$ & $<10$ & \\
\hline Mixed Comorbidity Index score, mean (SD) & 0.2 & 1.13 & 0.08 & 1.07 & 0.28 & 1.21 & 0.23 & 1.07 \\
\hline
\end{tabular}

Mixed Comorbidity Index score, n (\%) 
Table 1 Demographic and clinical characteristics at baseline (Continued)

\begin{tabular}{|c|c|c|c|c|c|c|c|c|}
\hline & \multicolumn{2}{|l|}{ All } & \multirow{2}{*}{$\begin{array}{l}\text { AGB } \\
\text { n/mean }\end{array}$} & \multirow[b]{2}{*}{$\% / S D$} & \multirow{2}{*}{$\begin{array}{l}\text { RYGB } \\
\text { n/mean }\end{array}$} & \multirow[b]{2}{*}{$\% / S D$} & \multirow{2}{*}{$\begin{array}{l}\text { SG } \\
\text { n/mean }\end{array}$} & \multirow[b]{2}{*}{$\% / S D$} \\
\hline & $\mathrm{n} /$ mean & $\% / \mathrm{SD}$ & & & & & & \\
\hline$<0$ & 1379 & $28.1 \%$ & 536 & $31.9 \%$ & 521 & $27.0 \%$ & 322 & $25.0 \%$ \\
\hline 0 & 1997 & $40.8 \%$ & 702 & $41.8 \%$ & 750 & $38.8 \%$ & 545 & $42.4 \%$ \\
\hline$>0$ & 1523 & $31.1 \%$ & 443 & $26.4 \%$ & 661 & $34.2 \%$ & 419 & $32.6 \%$ \\
\hline Hospital length of stay in days, mean (SD) & 0.73 & 4.31 & 0.39 & 2.14 & 1.14 & 6.03 & 0.58 & 3.15 \\
\hline
\end{tabular}

*Adapted from Flum et al. [26]

RYGB Roux-en-Y gastric bypass procedure, $A G B$ Adjustable gastric banding, SG Sleeve gastrectomy

$120(43.6 \%)$ had at least one admission in index health system. Overall, 761 out of 1361 (55.9\%) who had hospitalizations were admitted to the index CRN health system, and the other 600 patients did not return to the index health system during the follow-up (Fig. 2).

For hospital episodes, a total of 1250 or $43.2 \%$ of the 2893 hospitalizations occurred in the index CRN health system (Table 3 ), and $56.8 \%$ were admitted to hospitals outside of the index CRN health system. Hospital admissions to the index CRN health system were slightly more likely to occur right after the index surgery, $55.1 \%$ within 1 year and $45.7 \%$ within 3 years after index date. For the 701 hospitalizations for abdominal operations/interventions, 482 (68.8\%) occurred in the index CRN health system, and 219 (31.2\%) were outside of the index CRN health system. 91.6\% (642 out of 701) abdominal operations/interventions were performed within 1 year after index surgery and the likelihood of occurrence in index health system was relatively stable over time.

An overall mortality rate of $1.4 \%$ (67 deaths out of 4899 patients) was observed using health plan claims data, health plan enrollment data, and Social Security Administration data. Of those, $46.3 \%$ (31 deaths) occurred in hospitals based on hospital discharge billing records in claims. For the 31 in-hospital deaths, 21 (67.7\%) occurred at the index CRN health system, in which 8 occurred in the admission for the index bariatric surgery; and 10 (32.3\%) outside of index CRN health system.

\section{Incidence rates}

The incidence rates increased when the claims data was expanded from a single index CRN health system to the full HCARN claims from across health systems, as shown in Fig. 3: all-cause hospitalization, 11.0 (95\% Confidence Internal $[\mathrm{CI}]: 10.4,11.6)$ to 25.3 (95\% [CI]: 24.4, $26.3)$ visits per 100 patient-years; abdominal operations/ interventions, $4.2(95 \%[\mathrm{CI}]: 3.9,4.6)$ to $6.1(95 \%[\mathrm{CI}]$ : $5.7,6.6)$ visits per 100 patient-years; in-hospital death, $0.2(95 \%[\mathrm{CI}]: 0.11,0.27)$ to $0.3(95 \%[\mathrm{CI}]: 0.19,0.38)$ death per 100 patient-years.

\section{Discussion}

This observational study demonstrated the value of administrative claims data in enhancing the ability to follow patient outcomes across health systems longitudinally. In our study of patients undergoing the most common bariatric procedures, about $54 \%$ of subsequent all-cause hospitalizations, $31 \%$ abdominal operations or interventions, and $32 \%$ of in-hospital deaths occurred outside of the index CRN health system during the 5-year follow-up period. These events could be missed when data are only available from a single health system.

The estimated 30-day rate of composite adverse events based on the available administrative claims data in our study aligned well with prior work. In the PCORnet bariatric study, Arterburn et al. reported that $3.8 \%$ of their patients had composite adverse events within 30 days after bariatric surgery based on EHR data from PCORnet

Table $\mathbf{2}$ Incidence rates for 30-day composite adverse events

\begin{tabular}{|c|c|c|c|c|}
\hline \multirow{2}{*}{$\begin{array}{l}\text { Outcome Events } \\
(n=4752) \\
\text { 30d Composite Adverse Events }\end{array}$} & \multicolumn{2}{|c|}{ Index Health System } & \multicolumn{2}{|c|}{ All health systems in HCARN } \\
\hline & 183 & $3.85 \%$ & 198 & $4.17 \%$ \\
\hline Death & 10 & $0.21 \%$ & 11 & $0.23 \%$ \\
\hline Venous thromboembolism & 15 & $0.32 \%$ & 19 & $0.40 \%$ \\
\hline Percutaneous, operative, or endoscopic intervention & 162 & $3.41 \%$ & 174 & $3.66 \%$ \\
\hline Failure to discharge from hospital within 30 days & 19 & $0.40 \%$ & 19 & $0.40 \%$ \\
\hline
\end{tabular}

PCORnet National Patient-Centered Clinical Research Network, CRN Clinical Research Networks, HCARN HealthCore Anthem Research Network 
Table 3 Adverse events up to 5 years follow-up

\begin{tabular}{|c|c|c|c|c|c|c|}
\hline \multirow{2}{*}{$\frac{\text { Outcome Events }}{\text { Number of patients }}$} & \multicolumn{2}{|l|}{ Overall } & \multicolumn{2}{|c|}{ Within index Health System } & \multicolumn{2}{|c|}{ Outside index Health System } \\
\hline & 4899 & & & & & \\
\hline Follow up time, mean (median) in months & 28.0 & 23.2 & & & & \\
\hline \multicolumn{7}{|l|}{ All-cause hospitalization } \\
\hline$\geq 1$ hospital admission, $\mathrm{n}(\%)$ & 1361 & $27.8 \%$ & 761 & $15.5 \%$ & 780 & $15.9 \%$ \\
\hline Number of visits, total (row \%) & 2893 & $100 \%$ & 1250 & $43.2 \%$ & 1643 & $56.8 \%$ \\
\hline \multicolumn{7}{|l|}{ a) within 1 year } \\
\hline$\geq 1$ hospital admission, $\mathrm{n}(\%)$ & 705 & $14.4 \%$ & 442 & $9.0 \%$ & 313 & $6.4 \%$ \\
\hline Number of visits, total (row \%) & 1076 & $100 \%$ & 593 & $55.1 \%$ & 483 & $44.9 \%$ \\
\hline \multicolumn{7}{|l|}{ b) within 3 years } \\
\hline$\geq 1$ hospital admission, n (\%) & 1209 & $24.7 \%$ & 687 & $14.0 \%$ & 662 & $13.5 \%$ \\
\hline Number of visits, total (row \%) & 2359 & $100 \%$ & 1079 & $45.7 \%$ & 1280 & $54.3 \%$ \\
\hline \multicolumn{7}{|l|}{ Abdominal operations/interventions } \\
\hline$\geq 1$ hospital admission, n (\%) & 540 & $11.0 \%$ & 392 & $8.0 \%$ & 175 & $3.6 \%$ \\
\hline Number of visits, total (row \%) & 701 & $100 \%$ & 482 & $68.8 \%$ & 219 & $31.2 \%$ \\
\hline \multicolumn{7}{|l|}{ a) within 1 year } \\
\hline$\geq 1$ hospital admission, $\mathrm{n}(\%)$ & 500 & $10.5 \%$ & 368 & $7.7 \%$ & 154 & $3.2 \%$ \\
\hline Number of visits, total (row \%) & 642 & $100 \%$ & 449 & $69.9 \%$ & 193 & $30.1 \%$ \\
\hline \multicolumn{7}{|l|}{ b) within 3 years } \\
\hline$\geq 1$ hospital admission, n (\%) & 518 & $10.9 \%$ & 381 & $8.0 \%$ & 162 & $3.4 \%$ \\
\hline Number of visits, total (row \%) & 675 & $100 \%$ & 470 & $69.6 \%$ & 205 & $30.4 \%$ \\
\hline \multicolumn{7}{|l|}{ In-hospital Death } \\
\hline Number of death, total (row \%) & 31 & $100 \%$ & 21 & $67.7 \%$ & 10 & $32.3 \%$ \\
\hline
\end{tabular}

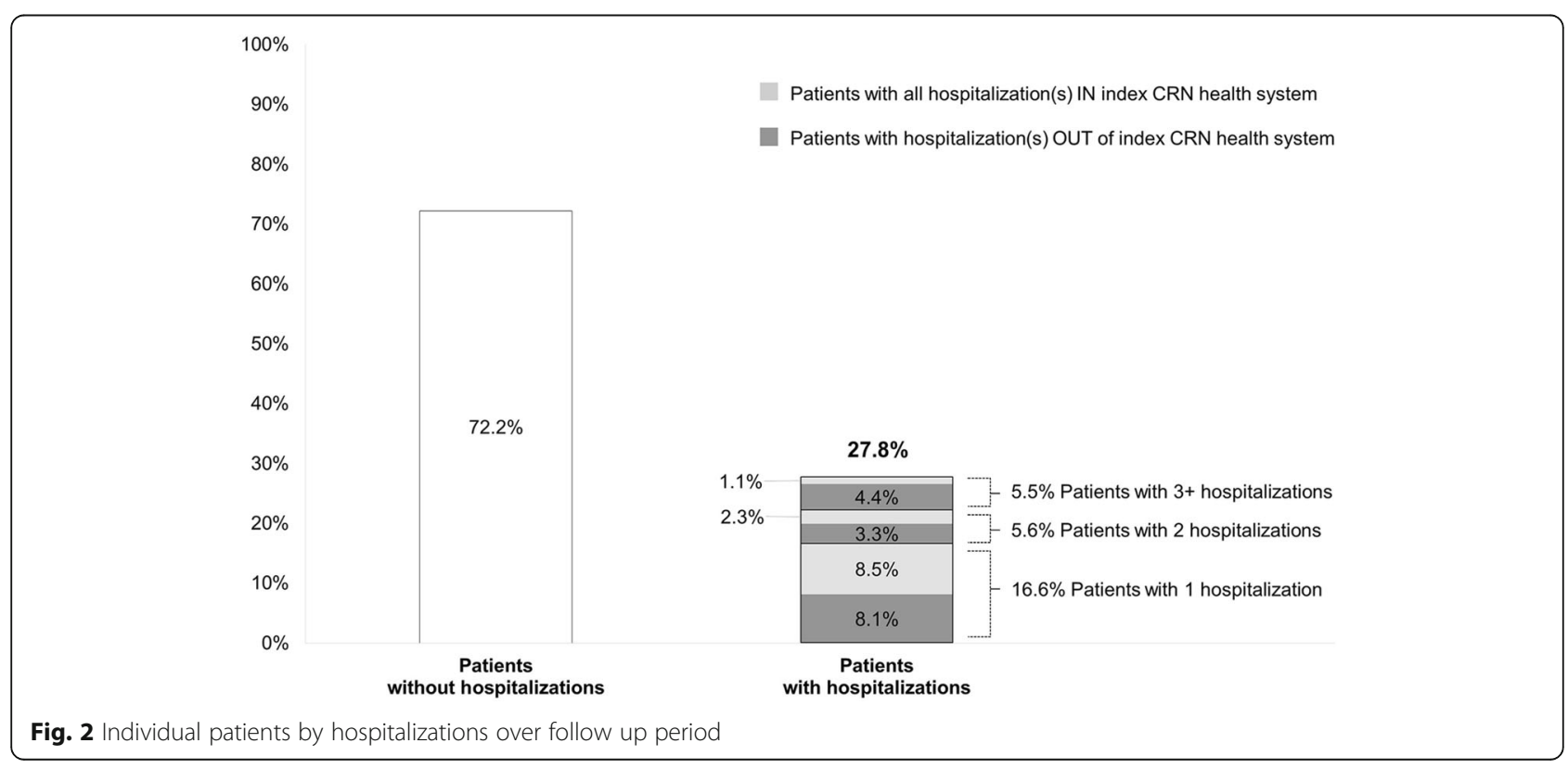


Incidence Rates (95\% Cl) per 100 Patient-Years

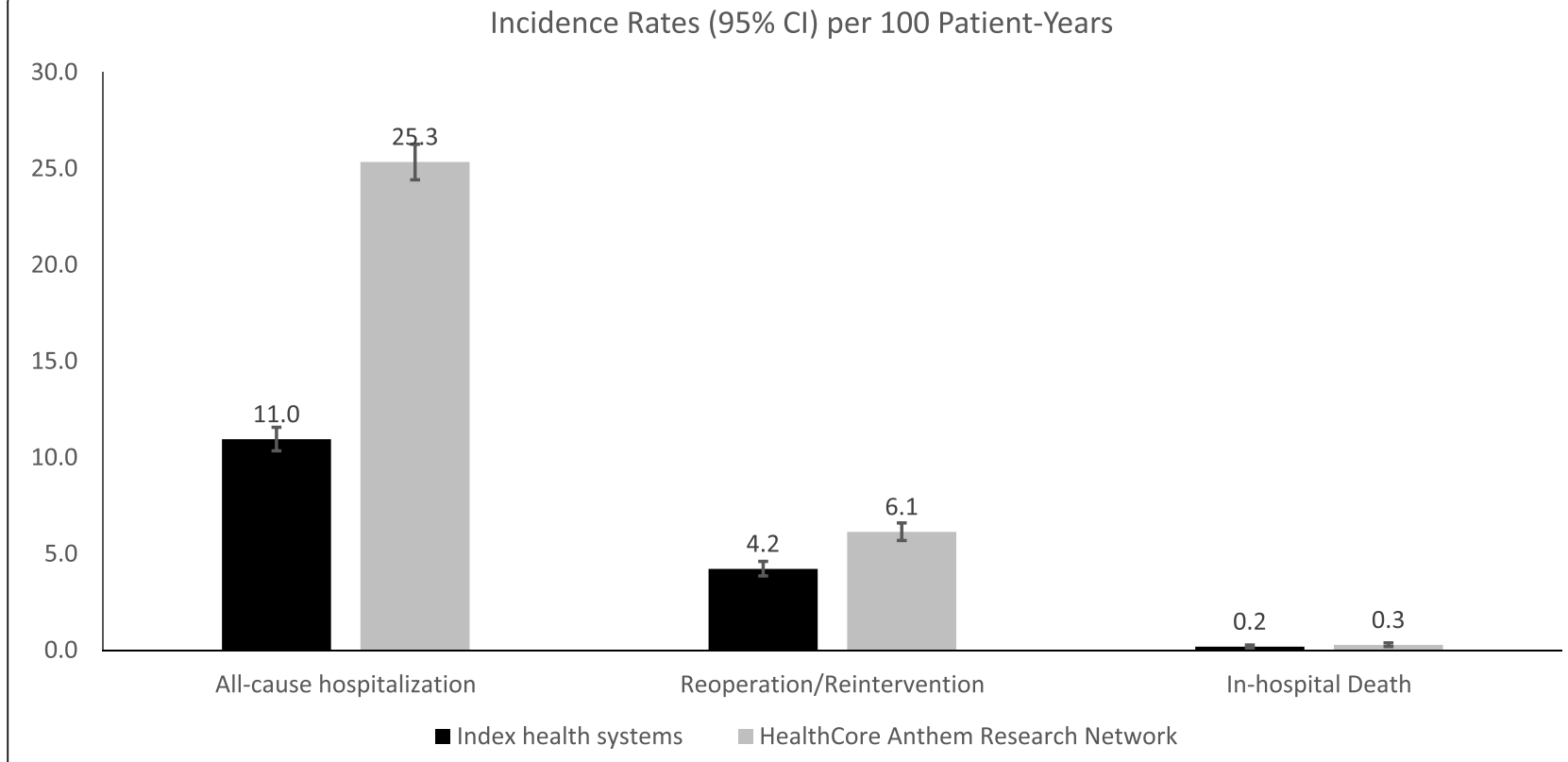

Fig. 3 Incidence rates of long-term adverse events

CRNs [22]. In our study, we observed rates of 3.9\% using data from PCORnet CRN health systems and 4.2\% using available data from HCARN, which were comparable to the Longitudinal Assessment of Bariatric Surgery (LABS) Study (4.1\% for composite averse events) [26].

The addition of claims data to outcome events was highly related to the timing of the event. The closer an event was to the index date, the likelier it occurred at an index CRN health system. For adverse events within 30 days, 183 of 198 (92.4\%) patients visited their index health system. For all-cause hospitalizations, the rate of index CRN health system admissions decreased from $55.1 \%$ in 1 -year follow-up to $45.7 \%$ in 3 -year follow-up. Abdominal operations or interventions in this study mainly occurred within 1 year after the index bariatric surgery, therefore, over time, approximately $69 \%$ of these procedures were at index CRN health systems.

Besides timing, the likelihood of receiving care at nonCRN health systems was related to the urgency of the event. For composite adverse events within 30 days, we observed that venous thromboembolism was more likely to occur outside of index CRN hospitals compared with surgical intervention (21.1\% of overall venous thromboembolism and $6.9 \%$ of overall surgical interventions occurred outside of index hospital). Patients with pulmonary embolism or deep vein thrombosis require urgent care and would be directed to the closest emergency room for immediate treatment. In contrast, if patients require a reintervention of a prior bariatric surgery, they might be more inclined to return to the health system where they had their initial surgery. In addition, our exploratory analysis that showed longer travel distance from patient's house to index hospital than to non-CRN hospital implied that location convenience of a health system played a role where patients are seeking urgent care.

One of the advantages of administrative claims data is the accuracy in measuring the enrollment time with informative censoring at time of disenrollment. Claims data have more accurate information on member censoring based on health plan enrollment records compared to EHR data, which often have incomplete information on when someone leaves a health system. This complete data capture of medically relevant events across health systems over defined periods of health plan enrollment is a strength of health plan claims data in longitudinal comparative effectiveness research.

Our results, on their own, and in conjunction with the findings of prior research that integrated clinical and claims data, have important implications for how treatment outcomes are tracked and managed across health systems. The value of claims data was demonstrated in our earlier PCORnet prospective longitudinal pragmatic study: Aspirin Dosing: A Patient-centric Trial Assessing Benefits and Long-Term Effectiveness (ADAPTABLE) trial designed to compare the effectiveness of two doses of aspirin. Applying the design of the ADAPTABLE trial in HCARN claims data, we found that claims data captured $>30 \%$ non-fatal and fatal hospital events in cardiovascular health occurring in health systems outside of a potential enrolling CRN health system [27]. Expansion of this approach could substantially increase the availability of information for clinical comparative effectiveness research. 
Of note, the combined EHR and claims data is a rare but emerging resource in the United States outside of integrated delivery systems. Data integration is difficult due to fragmented healthcare delivery. There are additional challenges on data standardization and governance. PCORnet has worked to close these gaps through application of data standardization and proposed data linkage governance. Our findings support the need for continued work to integrate data sources for complete capture of clinical outcomes in observational analyses. Our research demonstrates the increase in identifying outcomes over longer follow-up periods closing significant data gaps and thus improving real-world evidence generation.

\section{Limitations}

Administrative claims data lack clinical information, such as changes in weight, blood pressure, cholesterol, and diabetic glycemic control. Claims data is limited to data capturing medically attended events and data necessary to document coded billable encounters through the utilization of diagnosis, procedure, and medication dispensing codes. Our ability to accurately ascribe events to particular CRN health systems was limited on the completeness of tax identification numbers and linking their affiliations within known health systems. Administrative claims data may have coding inaccuracies, which might have introduced outcome misclassification into our study. In addition, patients included in this study were commercially insured and received care at PCORnet CRNs, consisting mainly of large academic medical centers in certain regions. Patients who seek care at these centers only reflect the utilization patterns in such institutions or geographic regions. Caution should be exercised when generalizing these results to different institutions, areas with less healthcare resources, or populations with different socioeconomic status.

\section{Conclusions}

Our study showed that patients receive care across health systems depending on the nature of the event such as timing, emergency, or location convenience. Administrative claims data provide a system for longitudinal ascertainment of health outcomes across health systems over defined enrollment periods within a health plan. These results suggest that supplementing EHR data with administrative claims data will capture a fuller picture of outcome events in longitudinal observational comparative effectiveness studies.

\section{Supplementary Information}

The online version contains supplementary material available at https://doi. org/10.1186/s12913-021-06074-3.
Additional file 1.

\section{Abbreviations}

PCORI: Patient-Centered Outcomes Research Institute; PCORnet: PatientCentered Clinical Research Network; HCARN: HealthCore Anthem Research Network; CRNs: Clinical Research Networks; HPRNs: Health Plan Research Networks; EHR: Electronic health records; AGB: Adjustable gastric banding; RYGB: Roux-en-Y gastric bypass; SG: Sleeve gastrectomy; CDM: Common data model; HIRD: HealthCore Intergraded Research Database; ICD: International Classification of Diseases; LABS: The Longitudinal Assessment of Bariatric Surgery Study; ADAPTABLE: Aspirin Dosing: A Patient-centric Trial Assessing Benefits and Long-Term Effectiveness

\section{Acknowledgements}

The authors thank Bernard B. Tulsi, MSc, Senior Medical Writer at HealthCore, Inc., for writing and other editorial support.

\section{Authors' contributions}

All the authors contributed substantially to the conceptualization and design of this study. Author's QM, MM, SS and KH, employees of HealthCore, were engaged in data acquisition. All of the authors, QM, MM, SS, KM and KH were involved in data analysis and interpretation, and participated in drafting and revising the different versions of this article. The published version of the article was approved by all authors.

\section{Funding}

This study was funded through a Patient-Centered Outcome Research Institute (PCORI) Award (HP-1510-32,545) for development of the National PatientCentered Clinical Research Network, known as PCORnet. PCORI had no role in approving the final manuscript.

\section{Availability of data and materials}

The datasets generated and analyzed during the current study are not publicly available due to containing information that could potentially identify study participants, but are available from the corresponding author on reasonable request.

\section{Ethics approval and consent to participate}

HealthCore is a wholly owned research subsidiary of Anthem with administrative permission to utilize Anthem data in accordance with strict compliance with the Health Insurance Portability and Accountability Act (HIPAA). This non-experimental study was performed under the Research Exception provisions of the Privacy Rule, 45 CFR 164.514(e), and was approved by the New England Investigational Review Board (IRB).

\section{Consent for publication}

Not applicable.

\section{Competing interests}

QM, MM, SS and KH disclose that they are employees of HealthCore, Inc., a wholly owned subsidiary of Anthem. KM participated in the bariatric study funded by the Patient-Centered Outcomes Research Institute (grant OBS1505-30,683).

\section{Author details \\ ${ }^{1}$ Translational Research for Affordability and Quality, HealthCore, Inc, Wilmington, DE, USA. 'ㄹepartment of Epidemiology, University of Pittsburgh, Pittsburgh, PA, USA.}

Received: 13 August 2020 Accepted: 12 January 2021

Published online: 04 February 2021

\section{References}

1. Fiks $A G$, et al. Comparative effectiveness research through a collaborative electronic reporting consortium. Pediatrics. 2015:136(1):e215-24.

2. Madden JM, et al. Missing clinical and behavioral health data in a large electronic health record (EHR) system. J Am Med Inform Assoc. 2016;23(6): $1143-9$. 
3. Bradley $\mathrm{CJ}$, et al. Health services research and data linkages: issues, methods, and directions for the future. Health Serv Res. 2010;45(5 Pt 2):1468-88,

4. Burton, L.C., G.F. Anderson, and I.W. Kues, Using electronic health records to help coordinate care. Milbank Q, 2004. 82(3): p. 457-81, table of contents.

5. Cifuentes $\mathrm{M}$, et al. Electronic health record challenges, workarounds, and solutions observed in practices integrating behavioral health and primary care. J Am Board Fam Med. 2015;28(Suppl 1):S63-72.

6. O'Malley AS, et al. Are electronic medical records helpful for care coordination? Experiences of physician practices. J Gen Intern Med. 2010;25(3):177-85.

7. Thompson, C.A., A.W. Kurian, and H.S. Luft, Linking electronic health records to better understand breast cancer patient pathways within and between two health systems. EGEMS (Wash DC), 2015. 3(1): p. 1127.

8. Desai $R$, et al. Comparative effectiveness of generic and brand-name medication use: a database study of US health insurance claims. PLoS Med. 2019;16(3):e1002763.

9. Hudson M, Tascilar K, Suissa S. Comparative effectiveness research with administrative health data in rheumatoid arthritis. Nat Rev Rheumatol. 2016;12(6): 358-66.

10. Kim SY, Solomon DH. Use of administrative claims data for comparative effectiveness research of rheumatoid arthritis treatments. Arthritis Res Ther. 2011; 13(5):129.

11. Popp RA, et al. Refining a claims-based algorithm to estimate biologic medication effectiveness and cost per effectively treated patient with rheumatoid arthritis. Pharmacotherapy. 2018;38(2):172-80.

12. Comparing the Benefits and Harms of Three Types of Weight Loss Surgery -The PCORnet Bariatric Study. Patient-Centered Outcomes Research Institute. https://www.pcoriorg/research-results/2015/comparing-benefits-and-harmsthree-types-weight-loss-surgery-pcornet-bariatric (accessed 08-17-2018).

13. Byrd JB, et al. Data quality of an electronic health record tool to support VA cardiac catheterization laboratory quality improvement: the VA clinical assessment, reporting, and tracking system for Cath Labs (CART) program. Am Heart J. 2013; 165(3):434-40.

14. Feder SL. Data quality in electronic health records research: quality domains and assessment methods. West J Nurs Res. 2018;40(5):753-66.

15. Johnson SG, et al. Application of an ontology for characterizing data quality for a secondary use of EHR data. Appl Clin Inform. 2016;7(1):69-88.

16. Esteban $\mathrm{S}$, et al. A rule-based electronic phenotyping algorithm for detecting clinically relevant cardiovascular disease cases. BMC Res Notes. 2017;10(1):281.

17. Boyer, A.P., et al., A Multilevel Approach to Stakeholder Engagement in the Formulation of a Clinical Data Research Network. Med Care, 2018. 56 Suppl 10 Suppl 1: p. S22-S26

18. Tambor E, et al. Engaging patients, clinicians, and the community in a clinical data research network: lessons learned from the CAPriCORN CDRN. Learn Health Syst. 2019;3(2):e10079.

19. Toh S, et al. The National Patient-Centered Clinical Research Network (PCORnet) bariatric study cohort: rationale, methods, and baseline characteristics. JMIR Res Protoc. 2017;6(12):e222.

20. Inge TH, et al. Comparative effectiveness of bariatric procedures among adolescents: the PCORnet bariatric study. Surg Obes Relat Dis. 2018;14(9):1374-86.

21. McTigue, K.M., et al., Comparing the 5-Year Diabetes Outcomes of Sleeve Gastrectomy and Gastric Bypass: The National Patient-Centered Clinical Research Network (PCORNet) Bariatric Study. JAMA Surg, 2020: p. e200087.

22. Arterburn D, et al. Comparative effectiveness and safety of bariatric procedures for weight loss: a PCORnet cohort study. Ann Intern Med. 2018;169(11):741-50.

23. Coleman KJ, et al. Understanding the bariatric patient perspective in the National Patient-Centered Clinical Research Network (PCORnet) bariatric study. Obes Surg. 2020;30(5):1837-47.

24. Courcoulas, A., et al., Interventions and operations 5 years after bariatric surgery in a cohort from the US National Patient-Centered Clinical Research Network Bariatric Study. JAMA Surg, 2020.

25. Hales, C.M., et al., Prevalence of Obesity and Severe Obesity Among Adults: United States, 2017-2018. NCHS Data Brief, 2020(360): p. 1-8.

26. Longitudinal Assessment of Bariatric Surgery, Flum DR, Belle SH, King WC, Wahed AS, Berk P, et al., Perioperative safety in the longitudinal assessment of bariatric surgery. N Engl J Med, 2009. 361(5): p. 445-54.

27. $\mathrm{Ma}$, Q., et al., Administrative claims data to support pragmatic clinical trial outcome ascertainment on cardiovascular health. Clin Trials, 2019: p. 1740774519846853.

\section{Publisher's Note}

Springer Nature remains neutral with regard to jurisdictional claims in published maps and institutional affiliations.

\section{Ready to submit your research? Choose BMC and benefit from:}

- fast, convenient online submission

- thorough peer review by experienced researchers in your field

- rapid publication on acceptance

- support for research data, including large and complex data types

- gold Open Access which fosters wider collaboration and increased citations

- maximum visibility for your research: over $100 \mathrm{M}$ website views per year

At BMC, research is always in progress.

Learn more biomedcentral.com/submissions 